\title{
A Hybrid Reconstruction Algorithm for Computerized Ionospheric Tomography
}

\author{
E. Yavuz', F. Arkan' ${ }^{2}$, O. Arikan ${ }^{3}$ \\ 'Finansal Teknoloji Hizmetleri A.Ş, eryavuz@fintek.com.tr \\ Ziraat Bankası Genel Müdürlügù, GMK Bulvan 162, Zemin Kat Ankara, Turkey \\ ${ }^{2}$ Hacettepe University \\ Department of Electrical and Electronics Engineering, arikan@hacettepe.edu.tr \\ Beytepe, Ankara Turkey \\ ${ }^{3}$ Bilkent Universiry \\ Department of Electrical and Electronics Engineering, oarikan@ee.bilkent.edu.tr \\ Bilkent, Ankara Turkey
}

\begin{abstract}
Computerized lonospheric Tomography (CIT) is a method to reconstruct ionospheric electron density images by using the Global Positioning System data coliected by the earth based receivers. In this study, Total Electron Content values abtained from a model based ionosphere and tomographic reconstruction techniques are used together to obtain ionospherie electron density distribution. Algebraic Reconstruction Technique (ART) is one of the most commonly used reconstruction method in medical tomography due to its simplicity in implementation. The performance of ART is independent of basis functions and very sensitive to the initial state. Total Least Squares (TLS) algorithm assumes no regularization and produces the lowest error for Haar basis for a given latitude interval. The performance of TLS is improved with the number of receivers. If only ane receiver is used, TLS algorithm together with Haar basis functions produces a low computational complexity and has a lower reconstruction error compared to Regularized Least Squares Algorithm. When the estimation by TLS is input as the initial state of ART, the overall reconstruction error reduces significantly compared to the reconstruction error of ART only or TLS with Haar basis only.
\end{abstract}

\section{INTRODUCTION}

Computerized Ionospheric Tomography (CIT) is a method to reconstruct ionospheric electron density images by using the Giobal Positioning System (GPS) data collected by the earth based receivers. GPS satellites transmit two simultaneous signals whose frequencies are $1575.42 \mathrm{MHz}$ and $1227.60 \mathrm{MHz}$. Total Electron Content (TEC) is defined as the number of free electrons in a column of unit crosssectional area [1]. TEC can be obtained from pseudo range and phase values recorded by the GPS receivers. TEC can also be obtained from model ionosphere density distribution such as International Reference lonosphere-95 (IRI-95) model by taking the line integral of electron density on the path combining the satellite and receiver. In ionospheric tomography, jonosphere is divided into pixels. Figure $l$ is a simplified example of ionospheric tomography system. Given in Figure $1, N_{k}$ indicates electron density in the pixel, $d_{k}$ indicates the length of ray in the $k$ th pixel. For these parameters, $k$ takes a value between $l$ and 4 , and TEC value computed at the receiver can be given as

$$
T E C=d_{c}\left(d_{1} \times N_{1}+d_{3} \times N_{3}+d_{4} \times N_{4}\right)+e_{r}
$$

where $d_{c}$ is an estimate constant and $e_{r}$ is the error term. In general, ionosphere electron density is modelled as a linear combination of two dimensional basis functions obtained by the product of the vertical and horizontal basis functions. Most commonly used horizontal basis functions are Legendre polynomials or Fourier polynomials [1-3]

The vertical basis functions are usually generated using the vertical profiles from a selected forward model, such as IRI95 [3]. Computational complexity of these methods is proportional the number of horizontal basis functions, so selection of appropriate number of horizontal basis function is a critical parameter.

In this paper, Total Least Squares (TLS) algorithm [5], Algebraic Reconstruction Technique (ART) [4], Hybrid Reconstruction Algorithm (HRA) which is combination of TLS and ART, and Regularized Least Squares (RLS) are discussed as a reconstruction algorithms.

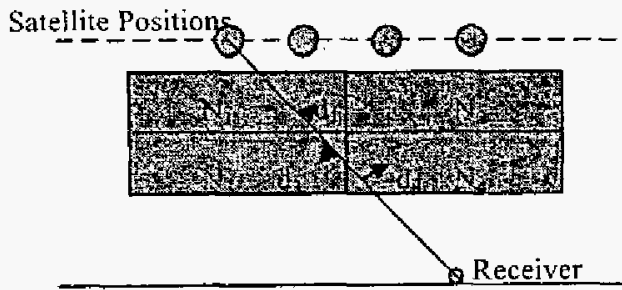

Figure 1. Sample Ionospheric Tomography System

Jonosphere electron density is modelled as the serial expansion method in which the two dimensional basis 
functions are used. Two dimensional basis functions are obtained from the vertical basis functions and horizontal basis functions. Verrical basis functions are generated from the Singular Value Decomposition (SVD) of the electron density matrix of forward ionosphere model (IRI-95) and horizontal basis functions are modelled from the Haar wavelets. It is observed that, due to the fact that TLS assumes no regularization, its computational complexity is less than the RLS, but RLS provides a more reliable result according to TLS. To improve the performance of TLS, ART is considered instead of the regularization method. The reconstructed electron density profile from TLS is input as the initial state of ART and a Hybrid Reconstruction Algorithm (HRA) is obtained. In HRA, although the computational complexity is increased compared to TLS alone due to addition of the ART algorithm. the implementation is still simpler and the overall error is lower than using ART or TLS alone.

\section{MODEL IONOSPHERE AND BASIS FUNCTIONS}

In this paper, IRI-95 is selected as a reference ionosphere model and ionosphere cross-section for $\left[-28^{\circ} 28^{\circ}\right]$ latiude interval is provided in Figure 2 using the parameter set given in TABLE I. Vertical basis functions given in Figure 3, are calculated by using the Singular Value Decomposition (SVD). Each vertical profile represents the variations according to height for a fixed latitude. Horizontal basis functions are selected as Haar basis functions given in Figure 4. Haar basis functions are mapped to $\left[-28^{\circ} 28^{\circ}\right]$ latitude interval and $\mathrm{x}$-axis is modelled as a distance between $-28^{\circ}$ and $28^{\circ}$ latitudes in which the one degree is equal to $111 \mathrm{~km}$.

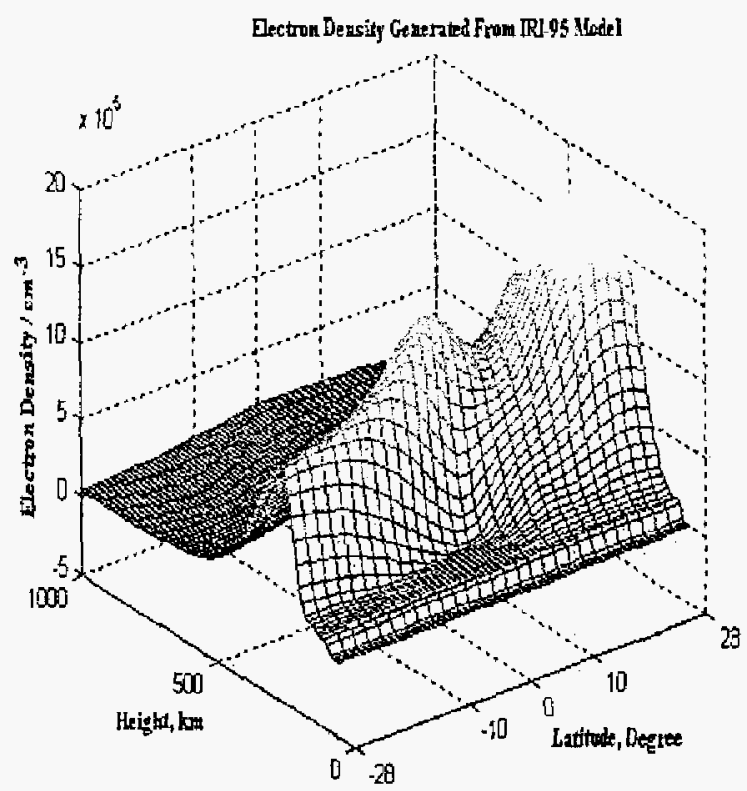

Figure 2 IRI-95 Model Parameters
TABLE I,

RI-95 MODEL PARAMETERS

\begin{tabular}{|l|l|}
\hline DATE: year, month, day & $2003,08,5$ \\
\hline Time: Hour & $15.5 \mathrm{LT}$ \\
\hline Geographical Lorgitude & 34 \\
\hline Solar Zenith Angle/degrec & 65.3 \\
\hline Dip (Magnetic Inclination)/degree & -60.62 \\
\hline Modip (Modified Dip)/degree & -48.14 \\
\hline Solar Sunspot Number & 52.3 \\
\hline lonospheric-Effective Solar Index IGI2 & 86.9 \\
\hline
\end{tabular}

In this study, ionosphere is divided into 95 pixels on the vertical direction and 29 pixels on the horizontal direction. Height of each pixel is $10 \mathrm{~km}$, and the width of each pixel is two degrees. It is assumed that the satellite travels on the flat line for a given latitude interval and the distance between each satellite position is equal to 0.5 degree. It is assumed that the ionosphere is time invariant for each sasellite positions and for each TEC calculations and electron density in each pixel has a uniform distribution.

\section{RECONSTRUCTION METHODS}

lonospheric electron density over height-latitude plane given in Figure 2, is expressed as a serial expansion of horizontal and vertical basis functions as given below:

$$
\begin{gathered}
g(r, \theta, N, M)=\sum_{k=1}^{K} \alpha_{k} \phi_{k}(r, \theta) \\
\phi_{k}(r, \theta)=u_{m}(r) v_{n}(\theta) \\
k=m+(n-1) M, m=1, \ldots, M ; n=1, \ldots, N
\end{gathered}
$$

where $\phi_{k}(r, \theta)$ is a two dimensional basis function and $u_{m}(r)$ is the vertical basis function obtained from IRI-95 model by SVD and $v_{n}(\theta)$ is the horizontal basis function chosen as Haar wavelets. In these expressions, $K$ is number of total basis functions, $M$ is number of vertical basis functions and $N$ is number of horizontal basis functions. $r$ is the height from sea level and it varies between $60 \mathrm{~km}$ to 1000 $\mathrm{km}$. Height is divided in $N R=95$ pixels, each of $10 \mathrm{~km} \theta$ is any angle between $-28^{\circ}$ to $28^{\circ}$ latitude. Latitude is divided in $N \Theta=29$ pixels each of $2^{*}$.

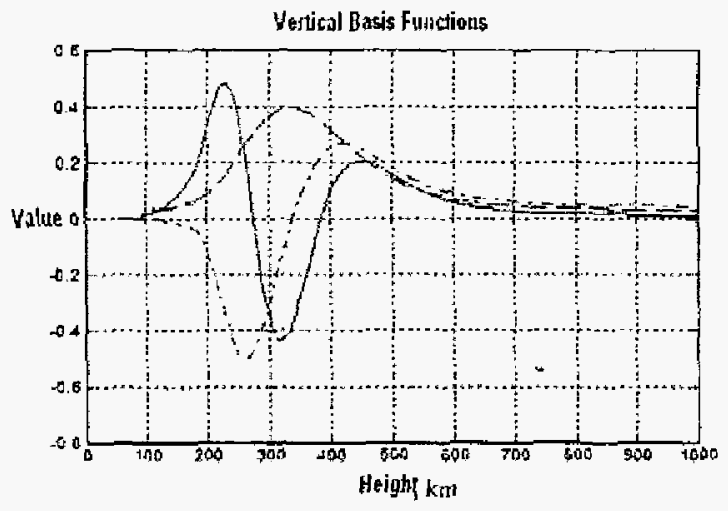

Figure 3. Vertical Basis Functions from IRI-95 Model. 

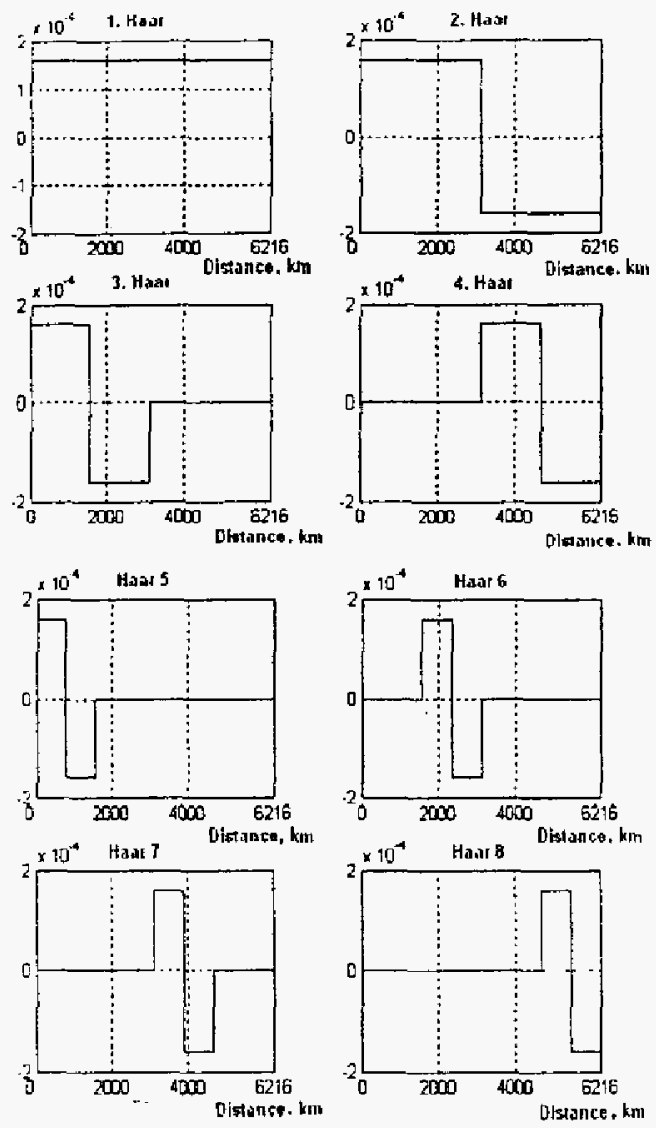

Figure 4. Haar Basis Funcrions.

Expression given in (4) is similar to the expression given in (1). In (1), equation is based on the TEC values and the expression given in (4) is based on the ionospheric electron density. Electron density, $g(r, \theta)$, is given in matrix notation in (6) and the two dimensional basis function for is also given in the matrix notation in (7).

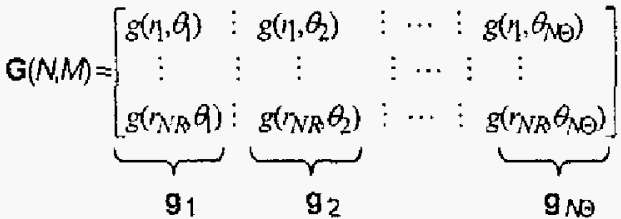

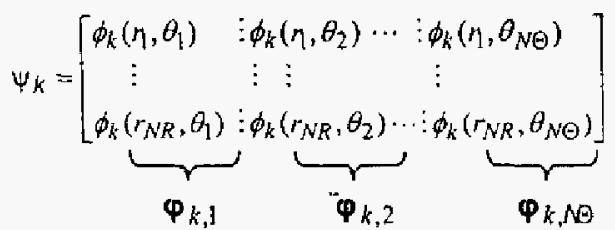

For simplicity, the calculations are continued with the vector form of $\mathbf{G}$ and $\psi$ given in (8).

$$
\mathbf{g}=\left[\begin{array}{c}
\mathbf{g}_{\mathrm{I}} \\
\ldots \ldots \\
\vdots \\
\ldots \ldots \\
\mathbf{g}_{N \Theta}
\end{array}\right] \boldsymbol{\varphi}_{k}=\left[\begin{array}{c}
\boldsymbol{\varphi}_{k, \mathrm{l}} \\
\ldots . . \\
\vdots \\
\ldots . . \\
\boldsymbol{\varphi}_{k, M O}
\end{array}\right]
$$

In vector $g, g\left(\mathrm{r}_{1}, \theta_{1}\right)$ represents the sample of electron density obtained from IRI-95 model at the height of $1000 \mathrm{~km}$ and the latitude of $-28^{\circ}$ degree. In CIT, TEC values are computed from GPS signals. In this study, TEC calculations are obtained from the scenario discussed in the previous sections using the IRI model. The measurement TEC vector can be expressed

$$
\begin{aligned}
& d_{l}=\sum_{p, q} \beta_{l, p, q} \mathbf{G}(p, q) \\
& \beta_{l, p, q}=\left\{\begin{array}{l}
1, \text { If }(p, q) \text { th pixel is on the } l \text { th ray. } \\
0, \text { Otherwise }
\end{array}\right.
\end{aligned}
$$

In (9), $l$ represents the index of ray from the satellite such as th ray (or index for satellite position, such as $l$ th satellite position) and $d_{l}$ is the TEC value which is calculated for /th ray (ith satellite position). (9) can be rewritten by using the vector, $\mathbf{g}$, as follows

$$
\begin{aligned}
& d_{l}=\sum_{k} \beta_{l, k} \mathbf{g}(k), \\
& \beta_{l, k}=\left\{\begin{array}{l}
1, \text { If }(p, q) \text { th pixel is on the } I \text { th ray }(10) \\
0, \text { Otherwise }
\end{array}\right. \\
& k=p+(q-1) M
\end{aligned}
$$

Equation (10) is given in matrix notation by using the scenario matrix $\mathbf{B}$ and the vector $\mathbf{g}$ as follow:

$$
\mathbf{d}=\mathbf{B} \mathbf{g}
$$

where $\mathbf{d}$ is the vector of TEC value from the measurement and is equal to $\mathbf{d}=\left[\begin{array}{lllll}d_{1} & d_{2} & d_{3} & \cdots & d_{L}\end{array}\right]^{T}, \mathrm{~L}=57$ is equal to total number of satellite positions. From the configuration of satellite and receiver positions, the scenario matrix, $B$, is calculated. The number of the colimns of the scenario matrix is equal to number of pixels in the horizontal direction and the number of the rows of the scenario matrix is equal to number of pixels in the vertical direction. The TEC calculation procedure is iterated for all satellite positions and elements of $B$ are assigned with respect w pirel siate given in grid geometry. For the given satellite position, all of the pixels on a satellite ray are determined and the elements of scenario matrix which corresponds to these pixels are assigned one and the other elements of scenario matrix are assigned zero. 
Equation (5) can be written in an inverse problem form as follows

$$
\mathbf{g}=\sum_{k} \mathbf{c}_{k} \boldsymbol{\Phi}_{k}
$$

Measurement equation given in (11) can be rewritten in the form of inverse problem by using the (12) as given below.

$$
\mathbf{d}=\mathbf{B} \mathbf{g} \cong \sum_{k} \mathbf{B} \varphi_{k} \alpha_{k}
$$

In (13), $a_{k}$ is the reconstruction coefficient and is calculated by using the reconstruction algorithm. This TEC calculation method is considered for all two dimensional basis functions to obtain the basis TEC values. In this step, all of the nwo dimensional basis functions are assumed to be subionosphere models, so the measurement equations from the basis TEC and the ionosphere TEC can be written in the form of (14) as

$$
\mathbf{d}=\mathbf{B} \mathbf{g}=\sum_{k} \mathrm{p}_{l, k} \alpha_{k}
$$

where $p_{l, k}$ is the vector contains the TEC values obtained from the $k$ th wo dimensional basis functions for the $l$ th satelite position. Each $\mathrm{P} /, k_{k}$ can be written in the form of

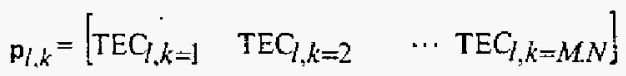

In $\mathrm{p}_{l, k}, \mathrm{TE} \mathrm{G}_{l, k=1}$ represents the obtained TEC value from the firsi rwo dimensional basis function for the $l$ th satellite position. The equation given in (14) is similar to (1). Each row of equations in (1) and (14) are based on the TEC values.

Equation (14) can be written in the matrix notation in (16) and the inverse problem equation for ionosphere tomography is obtained:

$$
\mathbf{d}=\mathbf{P} \mathbf{a}
$$

where

$$
\begin{gathered}
\mathbf{P}=\left[\begin{array}{llll}
\mathbf{p}_{1, k}^{H} & \mathbf{p}_{2, k}{ }^{H} & \cdots & \mathbf{p}_{l, k}^{H}
\end{array}\right] \\
\boldsymbol{\alpha}=\left[\begin{array}{lll}
\alpha_{1} \alpha_{2} & & \alpha_{M, N}
\end{array}\right] .
\end{gathered}
$$

In ionospheric tomography, it is desired to find an optimum coefficient set $a_{k}$ by using the reconstruction algorthms. In the following, the method of determining $a_{k}$ with the TLS is discussed. ART is also examined to find reconstructed image of ionosphere electron density.

\section{A. Total Least Squares (TLS) Algoriltht}

In the least-squares problem, the solution for (I6) is obtained with the assumption that the matrix $\mathbf{P}$ is correct, and any errors in the problem are in $\mathbf{d}$ which is projected onro the range of $P$ to find the solution. $B y$ the assumption, any changes needed to find a solution must come by modifying only d However, in ionospheric tomography problem, $\boldsymbol{P}$ is determined from the model TEC values, and may also have errors. Thus, it may be of use to find a solution to the problem given in (16) which allows for the fact that both $\mathbf{P}$ and $\mathbf{d}$ may be in error. Problerns of this sort are known as TLS problems.

In TLS problem, $\mathbf{d}$ and $\mathbf{P}$ are angumented with possible error vectors and matrices, respectively, and the solution of the perturbed equation

$$
(d+e)=(P+E) \alpha
$$

is investigated. Generally in applications of TLS, the number of equations exceeds the number of unknowns. However, TLS may also be applied to the problems in which the number of unknowns are more than the number of equations. In this case, an infinite solution set exists and the TLS solution method points the one with the minimum norm.

The closed-form TLS solution is given by the following

$$
\boldsymbol{\alpha}_{\mathrm{TLS}}=\left(\mathbf{P}^{H} \mathbf{P}-\sigma_{n+1}^{2} \mathrm{I}\right)^{-1} \mathbf{P}^{H} \mathbf{d}
$$

where $\sigma_{n+1}^{2}$ is the smallest non-zero singular value of the matrix $\mathbf{C}=[\mathbf{P} \mid \mathbf{d}], H$ represents Hermitian Transpose, and $\mathbf{I}$ is the identity matrix [5], [6], [7], [8], [9].

In [8], it is shown that when $P$ has full column rank, the TLS solution is related to the right singular vector $\mathbf{V}_{n+1}$ of $\mathbf{C}$ associated with the smallest singular value $\sigma_{n+1}$ under the condition that the singular value

$$
\sigma_{n}>\sigma_{n+1} \text { and } v_{n+1, n+1} \neq 0
$$

Here $\sigma_{n}$ represents the $n$th largest singular value and $v_{n+1, n+1}$ is the last component of the singular vector $\mathbf{V}_{n+1}$. Let $\mathbf{v}_{n+1}^{H}=\left[\widetilde{\mathbf{v}}_{n+1}^{H} v_{n+1, n+1}\right]$ where the $\widetilde{\mathbf{v}}_{n+1}^{H}$ is subvector that contains the first $N$ elements of $\mathbf{V}_{n+1}$. By using the $\widetilde{\mathbf{V}}_{n+1}$ and $v_{n+1, n+1}$, the TLS solution for the full-rank system is

$$
\alpha_{\text {TLS }}=-\frac{1}{v_{n+1, n+1}}-\tilde{\mathbf{v}}_{n+1}
$$

For rank-deficient systems and hence underdetermined systems, the TLS solution can be given as follows 


$$
\boldsymbol{\alpha}_{\mathrm{TLS}}=\frac{\sum_{i=r+1}^{n-1} v_{i, n+1} \widetilde{\mathbf{v}}_{i+1}}{\sum_{i=r+1}^{n+1} v_{i, n+1}^{2}}
$$

where

$$
\sigma_{p}>\sigma_{p+1}=\ldots=\sigma_{n+1}=0 \text { and } \mathbf{v}_{i}=\left[\widetilde{\mathbf{v}}_{i}^{H} v_{i, n+1}\right]^{H}
$$

are singular values and singular vector of $\mathbf{C}$, respectively. From the previous discussion, TLS solution can be obtained with the SVD of C [5], [6], [7], [8], [9].

\section{A. Algebraic Reconstruction Technique (ART)}

As shown in Figure 1, a square grid is superimposed on the image $g(r, \theta)$ and is assumed that the in each cell, $g(r, \theta)$ is constant. $g$, denotes this constant value in $j$ th ceil and the total number of cell in square grid is equal to $N$

From this geometry, TEC value for the ray can be written in the forn given below:

$$
\mathrm{TEC}_{\mathrm{i}}=\sum_{m=1}^{N} w_{\mathrm{i} m} g_{m}
$$

where upper bound for $i$ is equal to number of satellite position in interval of $\left[-28^{\circ} 28^{\circ}\right]$ latitude. $w_{i m}$ is the weight coefficient for the $m$ th pixel. In equation (22), $w_{i m}$ and $g_{m}$ are same as the parameters $d_{k}$ and $N_{k}$ given in (1). The measurement equation system for the algebraic reconstruction technique can be obtained by using (22).

The solution is calculated for the set of equations obtained by using the (22), but the initial value is needed as

$$
\mathbf{g}^{0}=\left[g_{1}^{0}, g_{2}^{0}, \ldots, g_{N}^{0}\right]
$$

In ionospheric tomography, iterative solution algorithm can be given as follows

$$
g^{i}=g^{i-1}-\frac{\left(g^{i-\uparrow} w_{i}-T^{-} C_{j}\right)}{w_{i} w_{i}} w_{i}
$$

where

$$
\begin{aligned}
& \mathbf{w}_{\mathbf{i}}=\left[w_{11}, w_{12}, \ldots, w_{1 N}^{*}\right] \\
& \mathbf{g}^{\mathrm{i}}=\left[g_{1}^{\mathrm{i}}, g_{2}^{\mathrm{i}}, \ldots, g_{N}^{\mathrm{i}}\right]
\end{aligned}
$$

This algorithm can also be expressed in a slightly different form:

$$
g_{j}^{\mathrm{i}}=g_{j}^{(\mathrm{i}-1)}+\frac{\left(\mathrm{TEC}_{\mathrm{i}}-\mathrm{TEC}_{\mathrm{i}}\right)_{w_{\mathrm{j} j}}}{\sum_{k=1}^{N} w_{\mathrm{i} k}{ }^{2}}
$$

where

$$
\begin{aligned}
\mathrm{TEC}_{\mathrm{i}} & =\mathbf{g}^{\mathrm{i}-1} \mathbf{w}_{\mathrm{i}} \\
& =\sum_{k=1}^{N} g_{k}^{(\mathrm{i}-1)} w_{i k}
\end{aligned}
$$

\section{B. Hybrid Reconstruction Algorithm (HRA)}

In this method, TLS algorithm and ART algorithm are considered together to obtain the tomograplic reconstruction of ionospheric electron density images. Here, the output of the TLS algorithm is used as the initial state for the ART.

TLS output can be written by using the $\boldsymbol{a}_{T L S}$ coefficients in the equation given in (12) as follows

$$
\hat{\mathbf{g}}_{\mathrm{TLS}}=\sum_{k} \mathrm{a}_{\mathrm{TLS}} \boldsymbol{\varphi}_{k}
$$

The reconstructed image obtained from (28) is used as the initial state of ART

$$
\mathrm{g}^{0}=\hat{\mathbf{g}}_{\mathrm{TLS}}
$$

and then the reconstruction is performed with the ART algorithm given in (24) to (26).

\section{RESULTS}

The optimum number of basis functions is an important parameter in performance of the reconstruction algorithms. Reconstruction error can be defined as

$$
\varepsilon(N, M)=\frac{\|\hat{\mathbf{G}}(N, M)-\mathrm{G}\|}{\|\mathbf{G}\|}
$$

where $G$ is electron density matrix obtained from IRI-95 model for $\left[-28^{\circ} 28^{\circ}\right]$ latitude interval, and $\hat{G}$ is the reconstructed electron density matrix. The error with respect to the number of horizonal basis funetion for TLS digurithn is given in Figure 5 for Haar Wavelets as horizontal basis functions. As can be observed from Figure 5 , the optimum number of horizontal basis functions for TLS algotithm is determined as the point where error drops to a value" whete increasing the number of basis functions do not reduce the error further. In TABLE II, the error norm, $\varepsilon$ (Nop, 3), for TLS with Haar and RLS with Cut- Legendre which is generated from the Legendre polynomial for the given latitude interval is given. Nop represents the optimum 
number of horizontal basis functions. As seen from the TABLE II, performance of RLS with Cut-Legendre is better than from the error of TLS with Haar, but the number of horizontal basis functions in TLS is lower than the number of horizontal basis functions in RLS, so the computational complexity is less in TLS. ART algorithm is independent of basis functions, so the computational complexity is important. In TABLE III, reconstruction error obtained with the ART alone and that of HRA are given. With the new approach, the performance of TLS is improved and the reconstruction error is comparable to that of using RLS algorithm alone. In HRA, the computational complexity is increased due to addition of the ART algorithm. Yet, implementation of ART is still simpler than that of RLS. The reconstructed image for HRA algorithm is given in Figure 6.

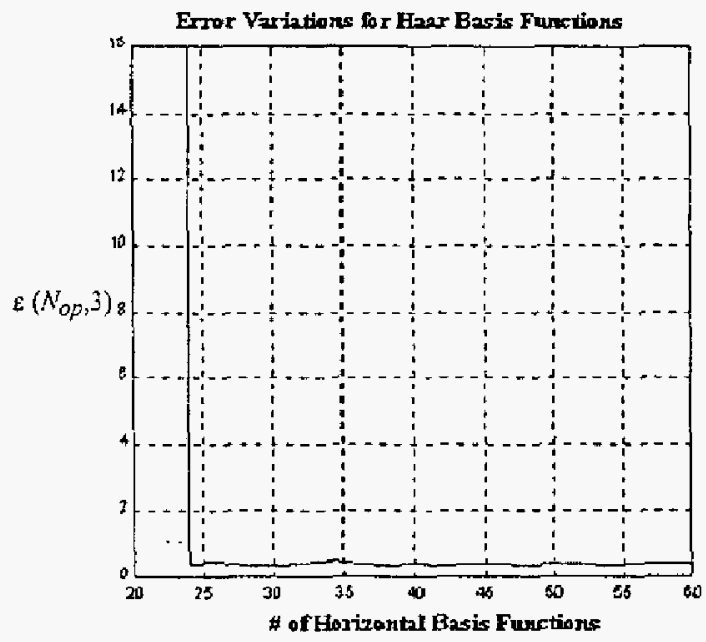

Figure 5. Error Variations for Haar Basis Functions in TLS

TABLE II

COMPARING THE PERFORMANCE OF TLS AND RLS

\begin{tabular}{|l|l|l|}
\hline & TLS + Haar & $\begin{array}{l}\text { RLS + Cut- } \\
\text { Legendre }\end{array}$ \\
\hline $\begin{array}{l}\text { hi of Horizontal Basis } \\
\text { Functions }\end{array}$ & 28 & 34 \\
\hline E.(Niop.3) & 0.1823 & 0.1798 \\
\hline
\end{tabular}

TABLE แI

ERROR NORM FOR RECONSTRUCTION ALGORITHMS

\begin{tabular}{|l|l|l|l|}
\hline & TLS + Haar & ART & HRT \\
\hline $\mathrm{c}\left(\mathrm{N} O P_{0} 3\right)$ & 0.1823 & 0.2279 & 0.1798 \\
\hline
\end{tabular}

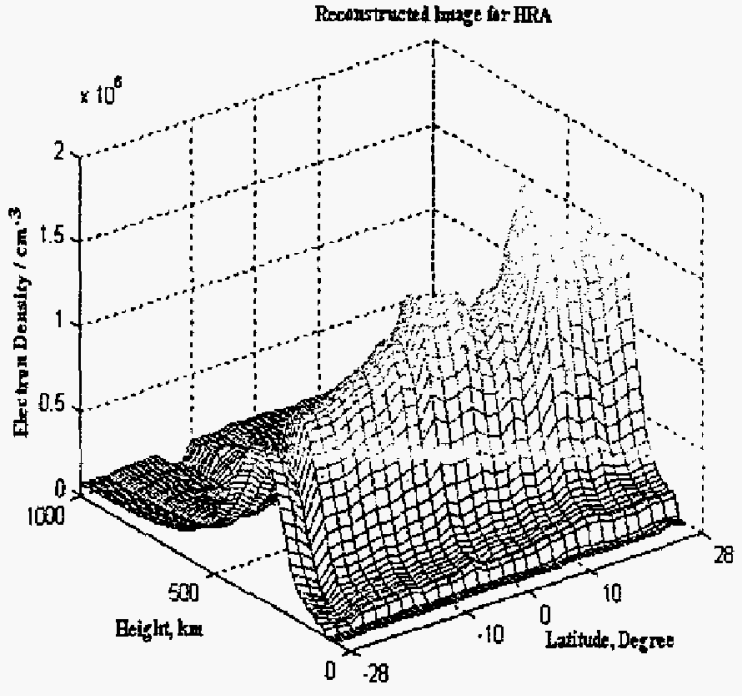

Figure 6. Reconstructed lmage for HRA

V. CONCLUSION

In this study, TLS algorithm with Haar basis function and ART algorithm is investigated for ionospheric tomography. TLS algorithm as used by itself, assumes no regularization and produces the lowest error for Haar basis for the given latitude interval. ART algorithm is independent of basis functions and very sensitive to the initial state. When the estimation by TLS is input as the initial state of ART, the overall reconstruction error reduces significantly compared to the reconstruction error of ART only or TLS with Haar basis only. The overall error for this scenario is comparable to using Regularized Least Squares algorithm together with Cut Legendre basis. Since the proposed method does not include any regularization, it is more efficient in implementation.

\section{V1. REFERENCES}

[1] R. A. Jeffrey, J. F Steven., C. H, Liu, "Iorospheric Irnaging Ustng Computerized Tomography". Radio Science, vol. 23, no. 3, pp. 299. 307, May/June 1988.

[2] E. Calais, J. B. Minster, "GPS, Earthquakes, The Lonosphere and the Space Shuttle", Physics of The Earth and Plametary Interiors, vol. 105, pp, 167-181, 1998.

[3] E. Sutton, H. Na, "High Resolution lonospheric Tomography Through Othogonal Decomposition", Proceedings of ICIP.94., IEEE International Conference, , vol. 2, pp. 148-152, 13-16 Nov. 1994

[4] A. C. Kak, M. Slaney, Principles of Compulerized Tomographic Imaging, JEEE Press, 1988.

[5] G. H. Golub, P. C. Hansen, D. P. O"Lcary. "Tikhonov regularization and total least squares", SIAM $J$. Matrix Anal Appl, vol. 21, pp. 185194. 2000.

[6] W. Zhu, Y. Wang and J. Zhang, "Total-Least Squares Recontruction with Wavelets for Optical Tomography", J. Opr. Soc. Am. A, wol. IS. no.10. Oct. 1998

[7] W. Zhu, Y. Wang, J. Zhang, J. Chang, H. L. Graber and R. I. Barbour, $Y$. Wang and J. Zhang, "lterative Total Least Squares Image Reconstruction Algorithm for Optical Tomography by The Conjugate Gradient Algorithm ", J. Opt. Soc. Am. A, vol. 14, no. 4, Apr. 1997.

[8] G. H. Golub and C. F: Van Loan, " Analysis of the Total Least Squares Problem ". SIAM J. Math Anal, wol. 17, pp 883-893. 1980.

[9] T. K. Moon, W. C. Stirling, Mathematical Methods and Algorithms for Signal Processing, Prentice Hall, 2000. 